\title{
Static digital telepathology: A model for diagnostic and educational support to pathologists in the developing world
}

\author{
Aliyah R. Sohani ${ }^{\mathrm{a}, *}$ and Moez A. Sohani ${ }^{\mathrm{b}}$ \\ ${ }^{a}$ Department of Pathology, Massachusetts General Hospital and Harvard Medical School, Boston, MA, USA \\ ${ }^{\mathrm{b}}$ The Fletcher School of Law and Diplomacy, Tufts University, Medford, MA, USA
}

\begin{abstract}
Background: The practice of pathology in the developing world presents challenges in terms of limited resources, shortages of trained personnel, and lack of continuing education programs. Telepathology holds promise as a means of diagnostic and educational support.

Methods: We donated multiheaded teaching microscopes equipped with digital cameras to four hospitals in Eastern Africa and trained local pathologists on their use. Static images of challenging cases were posted on a web-based telepathology platform. A U.S.-based pathologist reviewed images in consultation with subspecialist colleagues.

Results: Over a period of 40 months, 109 cases were submitted for second opinion consultation, including 29 dermatopathology cases (26.6\%), 14 hematopathology cases $(12.8 \%)$, and 13 cases each (11.9\%) in cytopathology and bone and soft tissue pathology. Static images enabled a complete or partial diagnosis in 100/109 cases (91.7\%). Factors precluding a definitive diagnosis included absence of confirmatory immunophenotyping, technical issues, or lack of clinical history. Case responses included a diagnosis and discussion, including differential diagnosis, references, and treatment recommendations.

Conclusion: Static digital telepathology is a simple, cost-effective, reliable and efficient means to provide diagnostic and educational support to pathologists in the developing world. Additional training may help overcome technical factors precluding a definitive diagnosis in certain cases.
\end{abstract}

Keywords: Telepathology, static imaging, developing world, teledermatopathology, telecytology

\section{Background}

The practice of pathology in the developing world presents numerous challenges in terms of limited resources, shortages of subspecialists and trained laboratory personnel, and lack of continuing education programs [1]. Retention of trained medical professionals in developing countries is further hampered by the quality of equipment and facilities and limited

*Corresponding author: Aliyah R. Sohani, MD, Department of Pathology, Massachusetts General Hospital, 55 Fruit Street, WRN 219, Boston, MA 02114, USA. Tel.: +1 617 726 3187; Fax: +1 617 643 6415; E-mail: arsohani@partners.org. opportunities to pursue advanced training and research. These challenges exist in a backdrop of a rising incidence of non-communicable diseases, including cancer, diabetes, and coronary artery disease. According to current estimates, $70 \%$ of newly reported cancers by the year 2030 will occur in the developing world, underscoring the importance of accurate pathologic diagnosis and staging to the health and well-being of the worldwide community [2].

Telepathology holds great promise as a means of offering diagnostic support, second opinion consultations, and ongoing training to pathologists practicing in resource-challenged settings [3]. Challenges in implementation of telepathology programs in the developing 
world center mainly around equipment cost; maintenance, servicing and repair; training of key users; high volume of data transmission; and internet connectivity, which may be limited in certain regions of the world. Despite their growing popularity in many pathology practices in the developed world for diagnosis, education and second opinion consultation, whole slide imaging systems may be impractical to implement in resource-challenged settings for many of the aforementioned reasons. On the other hand, static imaging systems present a potentially simpler and more cost-effective approach that is both reliable and sustainable, and that can be easily replicated across multiple settings. One potential drawback of static imaging systems is that they rely heavily on the referring pathologist to select appropriate fields for image capture and to obtain images of sufficient quality to facilitate a diagnosis. While this may be perceived as a disadvantage, the importance of maintaining a critical role for pathologists practicing in developing countries cannot be understated, particularly for programs whose mandates include education and capacity building.

We report our experience with a static digital telepathology program established to support a regional healthcare network of four hospitals in Kenya and Tanzania, affiliated with the Aga Khan University and the Aga Khan Development Network (AKDN). The largest hospital, located in Nairobi, Kenya, is an academic medical center staffed by five histopathologists that houses a pathology residency training program and an immunohistochemistry laboratory with a limited test menu [4]. This institution serves as a referral center for difficult and challenging cases to the three smaller affiliated hospitals located in Mombasa and Kisumu, Kenya and Dar es Salaam, Tanzania, whose pathology departments are each staffed by solo histopathologists.

\section{Methods}

Multiheaded teaching microscopes equipped with mounted digital cameras were donated and delivered to the four participating hospitals in East Africa. This was accomplished over a period of 18 months and involved site visits to the participating centers by a two-person team, including a pathologist and a consultant with expertise in both information systems and international affairs and development. During each site visit, local pathologists and other designated hospital staff, including laboratory managers and biomedical engineers, were provided with one-onone training on image acquisition using the camera and software, image upload to the telepathology website, and care and maintenance of the equipment, including a basic troubleshooting and repair guide. Static images were uploaded to one of two passwordprotected iPath telepathology servers (initially to http://telemed.ipath.ch/rahp hosted by Telemed Basel, Switzerland, then to http://ipath.aku.edu/rahp hosted by the AKDN eHealth Resource Centre, Karachi, Pakistan), which are equipped with an electronic mail alert system that lets participants in a user group know when a new case is posted or when comments are posted in response to a case $[5,6]$. The program's main objective was to provide both diagnostic and educational support to submitting pathologists. The same pathologist who participated in the site visits, upon returning to the United States, reviewed cases online in consultation with subspecialist colleagues and posted responses to cases with the goal of fulfilling this objective [7].

\section{Results}

Over a period of 40 months since the program was initiated, 109 cases have been submitted for second opinion consultation, including 29 dermatopathology cases (26.6\%); 14 hematopathology cases (12.8\%); 13 cases each $(11.9 \%)$ in cytopathology and bone and soft tissue pathology; eight cases each (7.3\%) in gastrointestinal, gynecologic, and head and neck pathology; and seven breast pathology cases (6.4\%) (Fig. 1). The remaining nine cases $(8.3 \%)$ comprised neuropathology, genitourinary, endocrine, pulmonary and medical renal pathology (Fig. 1). The 13 cytopathology cases included ten fine needle aspirations (FNAs), two fluid samples (one pleural fluid and one nipple discharge specimen), and one cervical smear. Pathologists from all four participating institutions posted cases, and $75 \%$ of consultations originated from one of the three smaller centers staffed by solo histopathologists (Fig. 2).

Each case contained an average of eleven images, including clinical images in eight cases (all dermatopathology cases), radiology images in four cases, and gross pathology images in four cases. Based on their review of the initial information and images posted, consulting pathologists had the option of asking for additional clinical history or requesting that 


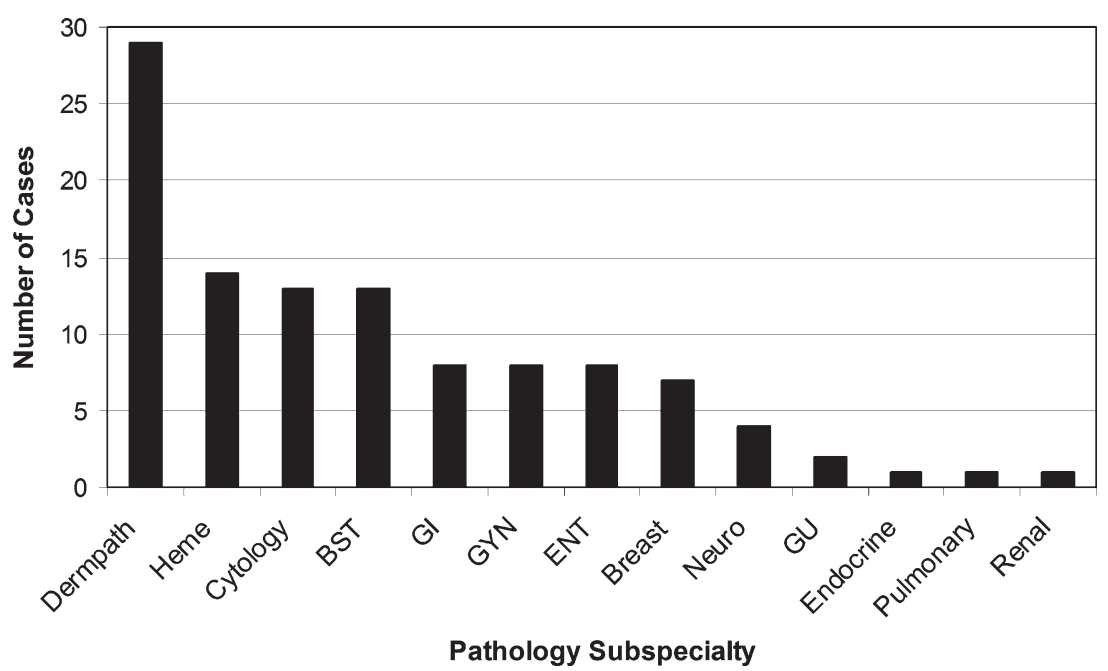

Fig. 1. Number of cases posted by pathology subspecialty. More than $60 \%$ of cases were posted in four subspecialty areas: dermatopathology (Dermpath), hematopathology (Heme), cytology, and bone and soft tissue (BST) pathology. Additional abbreviations: GI - gastrointestinal; GYN - gynecologic; ENT - ear, nose and throat; Neuro - neuropathology; GU - genitourinary.

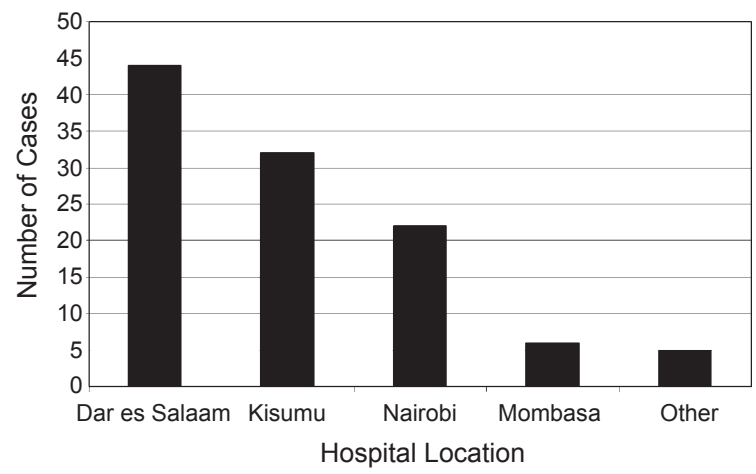

Fig. 2. Number of cases posted based on hospital of origin. The "Other" category refers to cases for which glass slides were sent by a referring hospital to one of the four participating institutions that had the telepathology equipment available for image capture and posting.

additional images be taken from certain fields of interest. Static images enabled a complete or partial diagnosis in 100/109 cases $(91.7 \%)$, and only nine cases $(8.3 \%)$ were entirely non-diagnostic. Among partially diagnostic and non-diagnostic cases, factors precluding a definitive diagnosis were related to absence of confirmatory immunohistochemistry or flow cytometry testing, lack of sufficient clinical history, or technical issues such as air-drying artifact, tangential tissue sectioning, poor staining or image quality, or inappropriate field selection. Insofar as such issues were related directly to the telepathology system, attempts to mitigate them were undertaken by providing the referring pathologist with tips and troubleshooting advice related to image quality via electronic mail or conference call. Most such issues were relatively minor, and no major equipment failures have been reported by any of the participating sites during the course of the program. In cases that required additional ancillary testing by immunohistochemistry or molecular genetic studies, we offered to evaluate the case in the traditional manner by requesting that glass slides or blocks be shipped to our institution. This occurred in only six instances $(5.5 \%$ of cases), and a definitive diagnosis or a narrow differential diagnosis was rendered in five cases using this approach.

Online responses posted to completely and partially diagnostic cases invariably had an educational emphasis: in addition to listing the favored diagnosis, they included a discussion of the differential diagnosis, the rationale for arriving at the favored diagnosis, diagnostic criteria, diagnostic pearls and pitfalls, and references relating to the favored entity or entities in the differential diagnosis, including published articles, websites, and gross and microscopic photographs. Treatment recommendations were provided in certain cases at the discretion of the consulting pathologist. The average turnaround time from initial posting of the case to posting of the final response was 5.6 days. 


\section{Discussion}

Our experience demonstrates that static digital telepathology is a simple, cost-effective, reliable and efficient means to provide both diagnostic and educational support to pathologists in the developing world. Feedback on our program from participating pathologists indicates that the technology is relatively easy to use and allows for fairly rapid access to second opinion consultation, without the need for shipping slides to referral centers in the vast majority of cases, thereby reducing turnaround time, as well as costs and risks of slide breakage. In addition, by implementing static imaging systems at four different hospitals in two countries in Eastern Africa, we were able to greatly enhance the number of physicians and patients served by digital pathology technology at a fraction of the cost of a single whole slide imaging system.

Despite its attractive cost and ease of use compared with robotic and whole slide imaging systems, static telepathology systems are subject to greater variability in terms of image quality and field selection bias [3]. However, our experience suggests that careful implementation with a strong educational focus may allow for certain advantages over more automated systems in terms of training and capacity building, by offering pathologists in the developing world a critical role in working up and maintaining ownership of a case. In particular, referring pathologists cited an increased sense of security on difficult cases, a greater awareness of certain diagnoses previously unknown to them, and the provision of references as key benefits of the program. We believe that the ultimate goal of implementing digital pathology programs in resource-challenged settings must be to build capacity and to create an enabling environment for referring pathologists to gain comfort and expertise in diagnostically challenging areas, in order to cope with the growing burden of noncommunicable diseases in the regions they serve [2].

Since this program's implementation, more than $60 \%$ of cases on which second-opinion consultation has been sought originate from four pathology subspecialty areas: dermatopathology, hematopathology, cytopathology, and bone and soft tissue pathology (Fig. 1). All four areas typically require fellowship training beyond residency in the United States; however, dedicated post-graduate fellowship programs in these areas are not available in Eastern Africa, suggesting the potential for programs of this kind to fulfill a critical unmet need in the region.
In particular, static digital telepathology appears well-suited to offering support for dermatopathology cases, which comprised more than a quarter of all cases for which second opinion consultation was sought (Fig. 1). This frequency exceeds the percentage of skin biopsies seen at the participating hospitals where, according to the submitting pathologists, such specimens account for only $5-15 \%$ of their volumes. The high rate of posting in this area is likely related to the complete absence of board-certified dermatopathologists in the region. In addition, the small size of typical skin biopsies minimizes limitations of field selection and sampling bias, increasing the likelihood of accurate digital diagnosis even when only selected fields are evaluated [8]. In a focused review of the 29 dermatopathology cases submitted for teleconsultation during our evaluation period, nearly two-thirds of the cases were inflammatory lesions, which are usually perceived by general pathologists as diagnostically more challenging than neoplastic lesions [9]. Glass slides available for review in $22 / 29$ cases $(76 \%)$ yielded a high diagnostic agreement of $95 \%$ between still image telepathology and glass slides diagnoses; the lack of agreement in the single discordant case was attributed to field selection bias (i.e., exclusion of the most diagnostic field of interest by the referring pathologist) (Gimbel DC et al., unpublished data). The dermatopathology cases have also included some rare and unusual entities, such as a case of concurrent Kaposi sarcoma and molluscum contagiosum diagnosed in a single biopsy [10]. Sharing of such cases at regional pathology meetings, and their subsequent publication in the peer-reviewed pathology literature, have provided educational and research opportunities to trainees and pathologists located at both the submitting and receiving institutions, an additional benefit of the telepathology program.

In contrast to dermatopathology, the subspecialty area of cytopathology appears to face greater challenges in offering diagnostic accuracy on a still image-based platform. Despite the relatively large number of cytopathology cases seen at the participating hospitals, and the fact that most cytology screening and evaluation is performed by histopathologists who do not have cytology subspecialty training, only about $12 \%$ of cases submitted for teleconsultation were cytology cases. In order to explore the reasons underlying this unexpectedly low rate of posting, we undertook a telecytology feasibility study, led by pathologists from both the United States and Eastern Africa [11]. Forty 
consecutive cytology cases, including cervical smears, fluids, and FNAs, were posted for review by one of the pathologists based in Eastern Africa and still images were evaluated by six regional colleagues with varying levels of experience in histopathology and cytopathology. Each pathologist's still image diagnoses were compared to consensus diagnoses generated by review of glass slides following a several month wash-out period. Diagnostic concordance rates between still image and glass slides diagnoses ranged from $71 \%$ to $93 \%$, and were higher for pathologists who had been in practice longer. More experienced pathologists also expressed greater levels of comfort with the technology and expressed higher levels of confidence in their digital diagnoses compared to their junior colleagues. In a large number of cases, causes of diagnostic discordance were related to poor image quality and field selection bias. Cytopathology specimens may be particularly challenging to review via digital means given the greater depth of focus required for their thorough evaluation, and the increased potential for sampling bias when cells are spread out over a large area of the slide and only certain fields are assessed [12, 13]. Despite these challenges, the findings of our feasibility study suggest that high rates of diagnostic accuracy may still be achieved with still image telecytology, provided that care is taken to minimize technical issues related to slide staining and smearing and that several photographs are taken from different areas of the slide. The level of experience of the consulting pathologist is also crucial, as pathologists who have been in practice longer appear to be more comfortable in rendering diagnoses on an entirely digital platform.

In summary, we present a simple and relatively inexpensive, yet efficient and reliable, model for diagnostic and educational support to pathologists in the developing world using a static imaging system and web-based case evaluation and reporting. Despite the known limitations of static imaging over whole slide imaging systems, we have maintained a relatively low frequency of non-diagnostic cases using this method and have achieved acceptable diagnostic concordance rates in instances in which glass slide review has been undertaken. When a definitive diagnosis has not been possible, limiting factors are mainly technical in nature and may be overcome by additional training on slide preparation, staining, and image capture to achieve higher quality images. When traditional glass slide evaluation has been required for a definitive diagnosis, we have used the telepathology system as a screening tool to select these cases sparingly, further minimizing costs and risks of slide breakage or loss during shipment. Due to its strong educational focus, the program has benefited referring and consulting pathologists alike, in terms of building confidence and achieving a greater sense of awareness of unusual entities, and has spawned several publications and ongoing research projects led by U.S. and East Africanbased pathologists in partnership with one another. The latter benefit has contributed to a more stimulating environment for intellectual discourse in a region in which retention of highly-trained personnel is an immense challenge. We encourage our pathology colleagues around the world who are fortunate enough to work in resource-rich environments to explore establishing similar partnerships as the one we describe with pathologists in resource-challenged settings. Collaborations such as these will serve to build global capacity for diagnostic excellence, a key component of any healthcare development strategy, thereby ensuring that patients throughout the world have access to accurate pathologic diagnoses as a critical part of their care.

\section{Acknowledgments}

The authors wish to thank the Aga Khan Health Board, USA, the Aga Khan Foundation, USA, and the Department of Pathology, Massachusetts General Hospital (MGH) for their support of the telepathology program; Telemed Basel and the AKDN eHealth Resource Centre for their support of the internet-based platforms that were used during this study; and all of the pathologists at MGH and other institutions who have generously donated their time and expertise in evaluating these cases.

\section{References}

[1] H. Benediktsson, J. Whitelaw and I. Roy, Pathology services in developing countries: A challenge, Arch Pathol Lab Med 131 (2007), 1636-1639.

[2] P. Farmer, J. Frenk, F.M. Knaul, et al., Expansion of cancer care and control in countries of low and middle income: A call to action, Lancet 376 (2010), 1186-1193.

[3] C.L. Hitchcock, The future of telepathology for the developing world, Arch Pathol Lab Med 135 (2011), 211-214.

[4] N. Kumar, S. Sayed, Z. Moloo and F. Rana, Global perspectives: The quest for knowledge in Cytopathology. Cytopathology Laboratory at the Aga Khan University Hospital in Nairobi, Kenya, American Society of Cytopathology Bulletin 47 (2010), 132-135. 
[5] K. Brauchli and M. Oberholzer, The iPath telemedicine platform, J Telemed Telecare 11 (Suppl 2) (2005), S3-S7.

[6] K. Brauchli, D. O'Mahony, L. Banach and M. Oberholzer, iPath - a Telemedicine platform to support health providers in low resource settings, Stud Health Technol Inform 114 (2005), $11-17$.

[7] A.R. Sohani, H. Chande and V. Vyas, Diagnostic and educational support to pathologists in the developing world through telepathology, Mod Pathol 22 (2009), 115A.

[8] D. Piccolo, H.P. Soyer, W. Burgdorf, et al., Concordance between telepathologic diagnosis and conventional histopathologic diagnosis: A multiobserver store-and-forward study on 20 skin specimens, Arch Dermatol 138 (2002), 53-58.

[9] D.C. Gimbel, A.R. Sohani, S.V.P. Busarla and R.M. Nazarian, Model for teledermatopathology in Africa: From concept to consultation (meeting abstract), Proceedings of the 47th
Annual Meeting of the American Society of Dermatopathology (2010).

[10] S.V.P. Busarla, S. Sayed, R.M. Nazarian, D.C. Gimbel, Z. Moloo and A.R. Sohani, Kaposi sarcoma in association with molluscum contagiosum: An uncommon diagnosis in a single biopsy and potential diagnostic pitfall, Am J Dermatopathol (2011), in press.

[11] N. Kumar, S.V.P. Busarla, S. Sayed, et al., Telecytology consultation in East Africa: A feasibility study of forty cases using a static imaging system, J Telemed Telecare (2011), in press.

[12] M.K. Baruah and F.G. Rosa, Optimal imaging in static telepathology, Indian J Pathol Microbiol 45 (2002), 367-370.

[13] L. Pantanowitz, M. Hornish and R.A. Goulart, The impact of digital imaging in the field of cytopathology, Cytojournal $\mathbf{6}$ (2009), 6. 


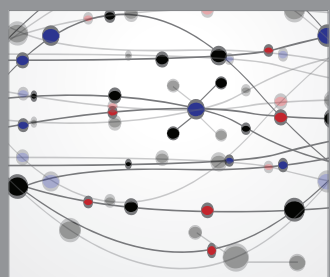

The Scientific World Journal
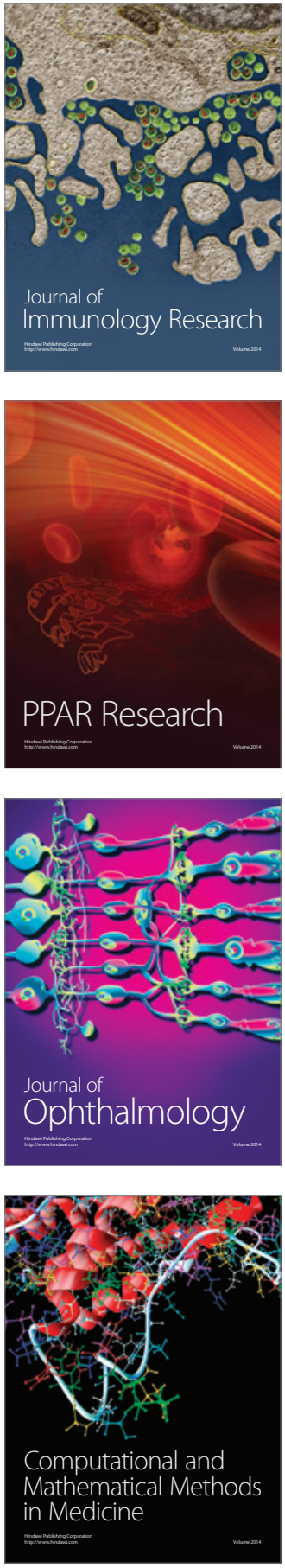

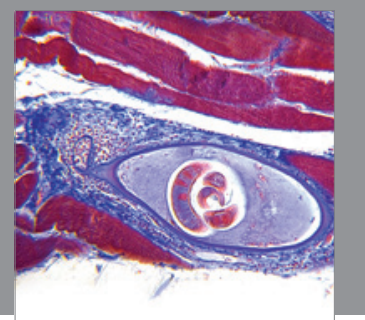

Gastroenterology

Research and Practice
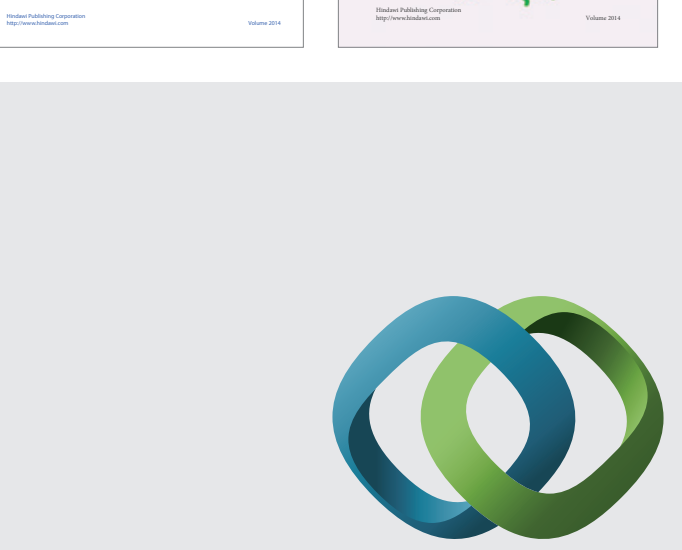

\section{Hindawi}

Submit your manuscripts at

http://www.hindawi.com
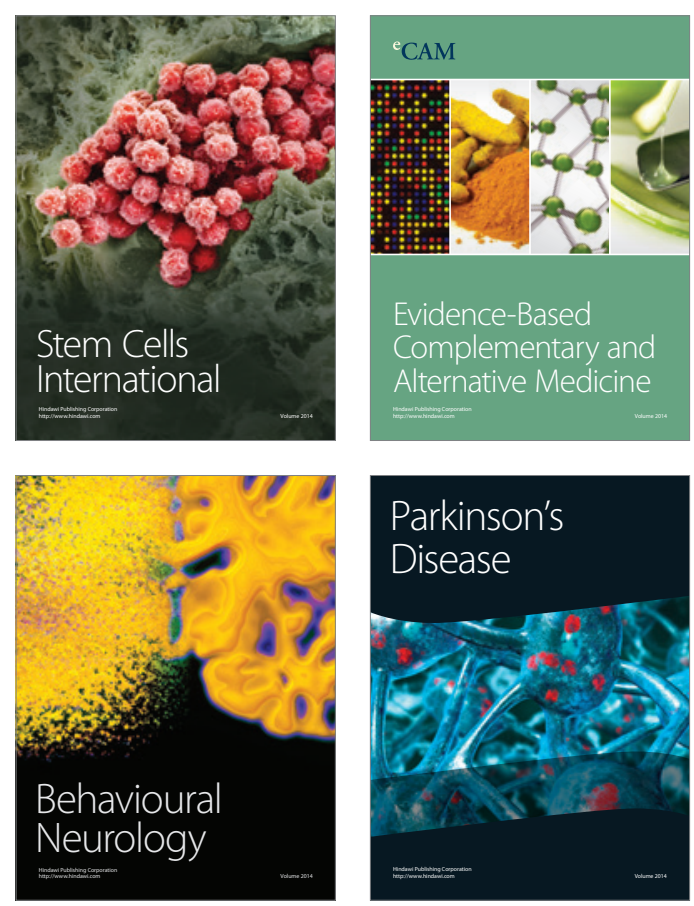

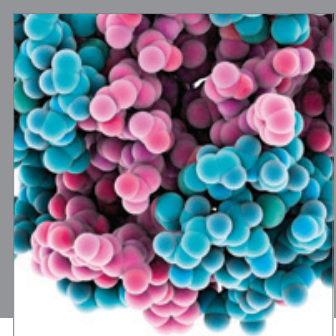

Journal of
Diabetes Research

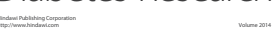

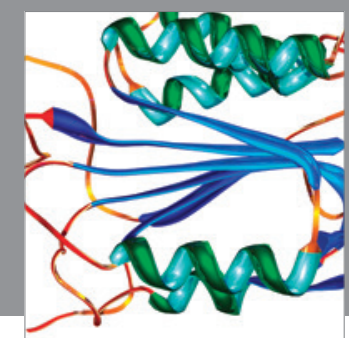

Disease Markers
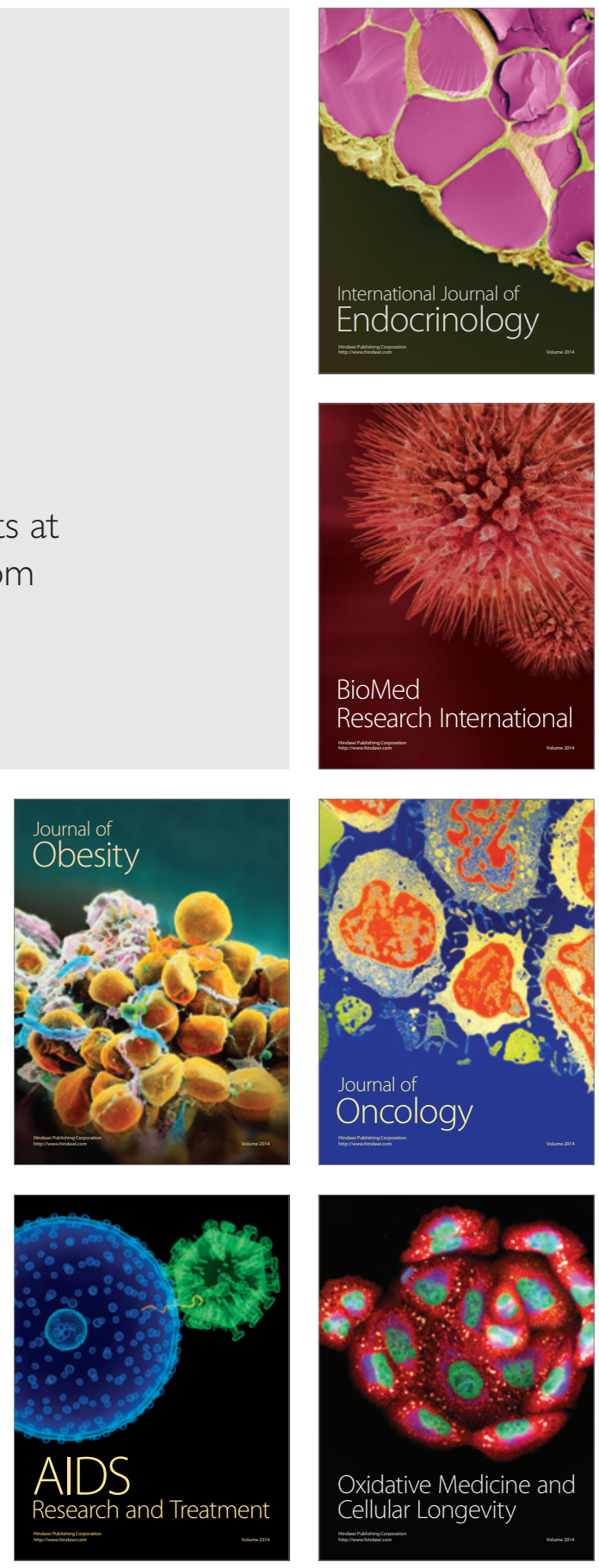